\title{
Performance Comparison of Multiple-Transmit Multiple-Receive V-BLAST algorithms
}

\author{
Hufei Zhu, Zhongding Lei and Francois Chin \\ Institute for Communications Research \\ 20 Science Park Road, Singapore 117674 \\ Email: engp1735@nus.edu.sg, \{leizd, chinfrancois\}@icr.a-star.edu.sg
}

\begin{abstract}
BLAST, a MIMO wireless communication systems can achieve very high spectral efficiency in rich multipath environment through exploiting the extra space dimension. A simplified version of BLAST known as V-BLAST has been proposed and implemented. In this paper we compare the performance of various V-BLAST algorithms through computer simulations. The results show that the nonlinear detection schemes with interference cancellation (IC) have much better performance than linear detection schemes and Minimum Mean Squared Error schemes (MMSE) have better performance than Zero Forcing (ZF) schemes. The efficient square-root algorithm with IC shows very attractive property that having low complexity while keeping the good performance compared with conventional IC scheme. In our simulation, little improvement is observed for the Pre-match filtering plus IC scheme.
\end{abstract}

Key Words V-BLAST, ZF, MMSE, IC, an Efficient square-root algorithm, Pre-MF

\section{Introduction}

Multiple-input multiple-output (MIMO) wireless communication systems can achieve very high spectral efficiency in rich multipath environment through exploiting the extra space dimension [1][2]. BLAST (Bell Labs Layered Space-Time) architecture [2][3] is such a system realizing very high data rates without additional power or bandwidth consumption. The diagonal BLAST or D-BLAST proposed by Foschini[4], utilizes multiple antenna at both transmitter and receiver and an elegant diagonally-layered coding structure in which code blocks are dispersed

The original version of this chapter was revised: The copyright line was incorrect. This has been corrected. The Erratum to this chapter is available at DOI: 10.1007/978-0-387-35618-1_37 
across diagonals in space-time. In an independent Rayleigh scattering environment, this processing structure leads to theoretical rates which increases linearly with the number of antennas (assuming equal numbers of transmit and receive antennas) with these rates approaching $90 \%$ of Shannon capacity. However, the D-BLAST suffers from low efficiency for short package transmission and it requires advanced encoding techniques and means to avoid catastrophic error propagation, which make it inappropriate for initial implementation.

In addressing these problems, a simplified version of BLAST known as vertical BLAST or V-BLAST has been proposed and implemented in real time in laboratory [3][5]. It is demonstrated that the V-BLAST is costeffective and highly spectrally efficient, achieving as much as up to $60 \%$ of the capacity achievable by D-BLAST. Therefore V-BLAST has drawn lots of attention recently and much effort has been made to achieve better performance [6][7] or lower complexity [8][9]. In this paper, we will compare the performance of various V-BLAST algorithms through computer simulations over Rayleigh fading channels.

This paper is organized as follows. The V-BLAST system is overviewed in section II followed by the description of various V-BLAST detection algorithms in section III. The simulation results are shown in section IV and concluded in section V.

\section{V-BLAST system overview}

The V-BLAST diagram is shown in Fig. 1 . This system consists of $\mathbf{M}$ transmitters and $\mathrm{N}$ receivers where $\mathrm{M} \leq \mathrm{N}$. At the transmitter end, a single data stream is de-multiplexed into $M$ streams, and each sub-stream is then encoded into symbols and fed to its respective transmitter.

The wireless channel is assumed to be rich-scattering and flat-fading. The fading between each antenna pair of transmitter and receiver is assumed independent.

At the receiver end, each receiving antenna receives the signals from all $\mathbf{M}$ transmitter antennas. Letting $\mathbf{a}=\left[a_{1}, a_{2}, \cdots, a_{M}\right]^{T}$ (' $\mathrm{T}$ ' denotes transpose operation) denotes the vector of transmit symbols from $M$ antenna, then the received signal can be represented as

$$
\mathbf{r}=\mathbf{H} \cdot \mathbf{a}+\mathbf{v}
$$

where $\mathbf{H}$ is the N-by-M complex channel matrix with statistically independent entries and $v$ is the complex Gaussian noise vector with zero mean and variance $\sigma_{v}{ }^{2}$. 

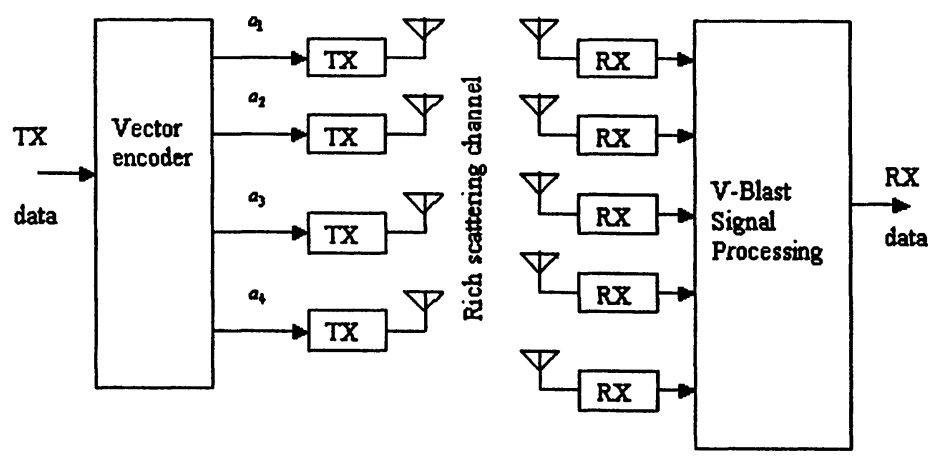

Figure 1: The block diagram of V-BLAST system

\section{DETECTION ALGORITHMS}

\subsection{Linear Detection}

Based on the formula (1), a linear detection is simply to multiply the received signal vector $\mathbf{r}$ with a linear transform matrix $\mathbf{G}$, i.e. the estimated signal vector may be represented as

$$
\widehat{\mathbf{a}}=\mathbf{G} \cdot \mathbf{r}=\mathbf{G} \cdot \mathbf{H} \cdot \mathbf{a}+\mathbf{G} \cdot \mathbf{v}
$$

This linear processing is also known as "nulling". Because the effect of the linear processing for each sub-stream is to keep the desired sub-stream signal while suppress or null the other sub-stream signals at the same time.

The linear detection algorithms differ from each other by the selection of $\mathbf{G}$, which is derived based on different criterion. The most common criteria for nulling are Zero Forcing (ZF) and Minimum Mean Squared Error (MMSE), for which the corresponding linear transform matrix are

$$
\begin{aligned}
\mathbf{G} & =\mathbf{H}^{+} \\
\mathbf{G} & =\left(\mathbf{H}^{H} \cdot \mathbf{H}+\boldsymbol{\sigma}_{\mathbf{v}}{ }^{2} \cdot \mathbf{I}_{M}\right)^{-1} \cdot \mathbf{H}^{H}
\end{aligned}
$$

respectively, where superscript ' + ', ' $H$ ', and ' -1 ' represent matrix pseudo inverse, Hermitian, and inverse operation respectively. As can be seen, the linear detection obtain estimates of all $\mathrm{M}$ signals at the same time. 


\subsection{Nonlinear Detection}

\subsubsection{Interference Cancellation (IC)}

Actually, the nonlinear detection schemes [1] with interference cancellation (IC) are borrowed from the multiuser detection context. This kind of detection methods does not detect the $M$ signals at one run. Instead, it starts with linear detection of only one sub-stream by means of nulling with ZF or MMSE, i.e. multiply $\mathbf{r}$ with a row vector of $\mathbf{G}$ instead of the matrix G. This sub-stream was selected because it was the best one amongst the rows, in the sense that it gets highest post-detection signal-to-noise ratio (post-SNR). Then the effect of the detected signal is subtracted out from the received signal vector, resulting in a modified received vector with less "interferers". This process proceeds until all the signals are detected.

Let the ordered set

$$
\varsigma \equiv\left\{k_{1}, k_{2}, \cdots, k_{M}\right\}
$$

be a permutation of the integers $1,2, \cdots, M$ specifying the order in which components of the transmitted symbol vector a are extracted. The full detection algorithm (with $\mathrm{ZF}$ nulling) can be described compactly as a recursive procedure, including determination of the optimal ordering for selection of the best row, as follows:

a) initialization:

$$
\begin{aligned}
& i \leftarrow 1 \\
& \mathbf{G}_{1}=\mathbf{H}^{+} \\
& k_{1}=\underset{j}{\arg \min }\left\|\left(\mathbf{G}_{1}\right)_{j}\right\|^{2}
\end{aligned}
$$

b) recursion

$$
\begin{aligned}
& \mathbf{w}_{k_{i}}=\left[\left(\mathbf{G}_{i}\right)_{k_{i}}\right]^{T} \\
& y_{k_{i}}=\mathbf{w}_{k_{i}}^{T} \cdot \mathbf{r}_{i} \\
& \hat{a}_{k_{i}}=Q\left(y_{k_{i}}\right) \\
& \mathbf{r}_{i+1}=\mathbf{r}_{i}-\hat{a}_{k_{i}} \cdot(\mathbf{H})_{k_{i}} \\
& \mathbf{G}_{i+1}=\mathbf{H}_{\bar{k}_{i}}^{+} \\
& k_{i+1}=\underset{j \notin\left\{k_{1} \cdots k_{i}\right)}{\arg \min }\left\|\left(\mathbf{G}_{i+1}\right)_{j}\right\|^{2} \\
& i \leftarrow i+1
\end{aligned}
$$

where $\left(\mathbf{G}_{i}\right)_{j}$ denotes the $j$ th column of $\mathbf{G}_{i}, \mathbf{H}_{\bar{k}_{i}}$ stands for the matrix obtained by zeroing columns $k_{1}, k_{2}, \cdots, k_{i}$ of $\mathbf{H},\|\cdot\|$ is the length of the 
vector and $Q($.$) denotes the quantization (slicing) operation appropriate to$ the constellation in use.

For nonlinear IC detection with MMSE, the steps are the same except $\mathbf{G}_{i},(i=1, \ldots, M)$ are calculated based on formula (4) instead of (3) above.

\subsubsection{An efficient square-root algorithm for IC (SRIC)}

An efficient square-root algorithm for nonlinear IC detection has been reported in [9]. This algorithm is to find an efficient way to compute the pseudo inverse in steps $(6 \mathrm{~b}) /(6 \mathrm{i})$ to ease computation burden. Actually it reuses the intermediate computation results of previous iterations for the current iteration to avoid re-computing the pseudo-inverse (or $Q R$ decomposition) for each deflated subchannel matrix.

\subsubsection{Pre-match filtering (Pre-MF) with IC}

The performance of IC detector for VBLAST varies with the channel matrix H. It may be improved through changing the distribution of the channel matrix. Pre-MF with IC scheme has been proposed based on this idea [10]. Before applying IC algorithm, it pre-process the channel matrix with a matching filter and fed the resulting cross-correlation matrix to the conventional BLAST IC detector for BPSK modulated signal. The signal after matched filter can be represented as

$$
\mathbf{Y}=\mathbf{H}^{H} \cdot \mathbf{r}=\mathbf{H}^{H} \cdot \mathbf{H} \cdot \mathbf{a}+\mathbf{H}^{H} \cdot \mathbf{v}=\mathbf{R} \cdot \mathbf{a}+\tilde{\mathbf{v}}
$$

where $\mathbf{R}=\mathbf{H}^{H} \mathbf{H}$ is the cross-correlation cahnnel matrix. Therefore the matrix $R$, replacing $H$, is used to go through the process of IC scheme. (from (6a)to (6k)).

\section{Simulation results}

In this section, we evaluate various detection algorithms over Rayleigh fading channel in the presence of white Gaussian noise through simulations. The algorithms are linear ZF, linear MMSE, IC with ZF, SRIC with ZF, SRIC with MMSE, pre-MF IC with ZF.

We consider the systems with 4 transmitter and 4 receiver antennas. Figure 2 shows cumulative distribution functions of SNR of these algorithms when pre-detection SNR is $10 \mathrm{db}$, while figure 3 shows corresponding cumulative distribution functions of channel capacity of these algorithms. The average BER (bit error rate) curves vs pre-detection SNR are shown in figure 4. 
From these figures we can see consistently that nonlinear detection schemes with IC perform much better than linear detection schemes in terms of post-detection SNR, capacity, and BER, but at the cost of more computational load. MMSE detection schemes (either linear or nonlinear) have better performance than their ZF counterparts because of taking into consideration of the effect of noises. It is notable that the SRIC algorithms have almost no performance degradation compared with their counterparts while keeping computational load quite low. As to pre-MF IC scheme, little improvement is observed compared with conventional IC scheme. This may be due to the assumption we made in our simulations that most of decisions for the first data stream are correct to ease computation load.

\section{Conclusion}

We present six V-BLAST algorithms. Simulation results show that the nonlinear detection schemes with IC has much better performance than linear detection schemes and MMSE has better performance than ZF. There is no obvious difference observed between the performance of the efficient square-root algorithm with conventional IC while the former has attractive low computing complexity. And little performance improvement is observed for the Pre-MF with IC scheme compared with conventional IC.

\section{Reference}

[1] P.W. Wolniansky, G.J. Foschini, G.D. Golden and R.A. Valenzuela, "V-BLAST: an architecture for realizing very high data rates over the rich-scattering wireless channel", in Proceeding of URSI International Symposium on Signals, Systems, and Electronics (ISSSE'98), pp. 295 -300, 1998.

[2] G.J. Foschini and M.J. Gans, "On limits of wireless communications in a fading environment when using multiple antennas", Wireless Personal Communications, vol. 6, no. 3, pp. 311-335, Mar. 1998.

[3] G.J. Foschini, "Layered space-time architecture for wireless communication in a fading environment when using multi-element antennas", Bell Labs Tech. J., pp.41-59, Autumn 1996.

[4] G.G. Raleigh and J.M.Cioffi, "Spatio-temporal coding for wireless communication", IEEE Trans. Commun., vol. 46, pp. 357-366, Mar. 1998.

[5] G.D. Golden, G.J. Foschini, R. A. Valenzuela, and P.W. Wolniansky, "Detection algorithm and Initial Laboratory Results using the V-BLAST Space-Time Communication Architecture”, Electronic Letters, Vol. 35, No. 1, Jan. 7, 1999, pp. 14-15. 
[6] Li, X.; Huang, H.; Foschini, G.J.; Valenzuela, R.A., "Effects of iterative detection and decoding on the performance of BLAST", Global Telecommunications Conference, 2000. GLOBECOM '00. IEEE, Volume: 2, 2000 Page(s): 1061-1066 vol. 2.

[7] Won-Joon Choi; Negi, R.; Cioffi, J.M. "Combined ML and DFE decoding for the VBLAST system", in Proceeding of IEEE International Conference on Communications, Vol. 3, pp. $1243-1248,2000$.

[8] Wong Kwan Wai; Chi-Ying Tsui; Cheng, R.S. "A low complexity architecture of the VBLAST system", Wireless Communications and Networking Confernce, 2000. WCNC. 2000 IEEE, Volume: 1, 2000 Page(s): 310-314 vol.1.

[9] Babak Hassibi "An efficient square-root algorithm for BLAST".

[10] S. Sfar, N. Boubaker, R.D. Murch, and K.B. Letaief, "Performance of packetized layered space-time detection over wireless links”, ISCC' 2001, pp592-596, 2001.

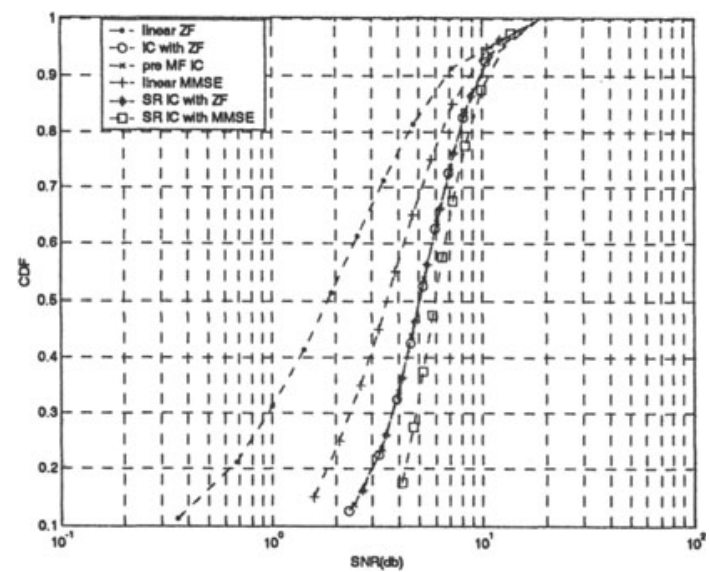

Figure 2: Cumulative distribution functions of SNR 


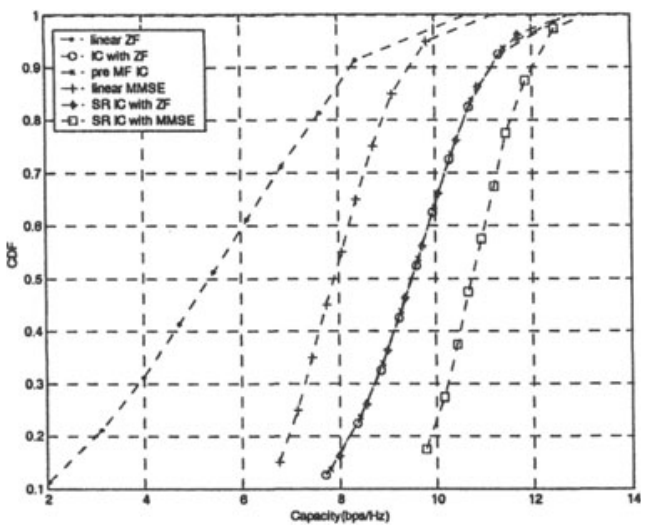

Figure 3: Cumulative distribution functions of channel capacity

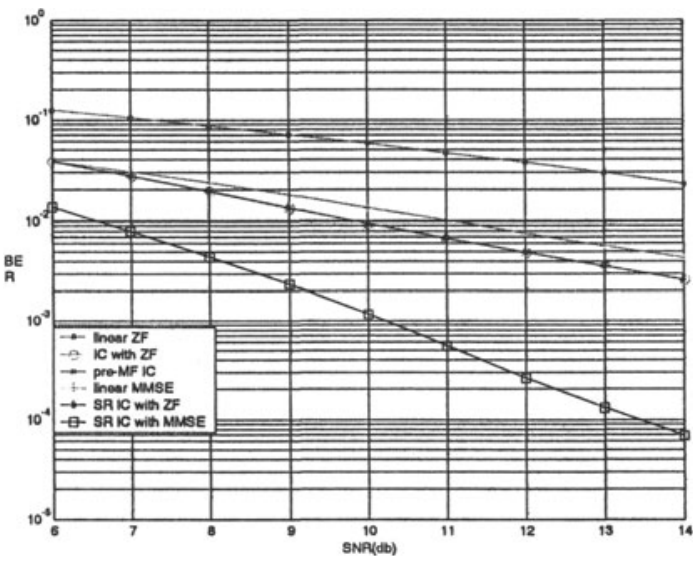

Figure 4: Average BER 